\title{
LQG control of linear lossless positive-real systems: the continuous-time and discrete-time cases
}

\author{
Maide Bucolo ${ }^{1,2} \cdot$ Arturo Buscarino $^{1,2}$ (D) Luigi Fortuna ${ }^{1,2} \cdot$ Mattia Frasca $^{1,2}$
}

Received: 24 May 2021 / Revised: 5 August 2021 / Accepted: 6 August 2021 / Published online: 8 September 2021

(c) The Author(s) 2021

\begin{abstract}
Lossless positive-real systems have been widely studied in the literature. They are systems in which the energy is entirely transferred between input and output. In this paper, new aspects related to the linear quadratic gaussian (LQG) control of lossless positive-real systems are reported including both the continuous-time and the discrete-time cases. Direct formulas for the calculation of the optimal gains will be introduced and the properties of the different structures of the LQG compensator obtained for the continuous-time and the discrete-time cases will be emphasized, also in view of designing positive-real LQG compensators. Numerical examples related to low-damped structures are also discussed to verify the possibility to design the LQG compensator on the basis of a lossless approximation.
\end{abstract}

Keywords Lossless systems · Optimal control · Weakly-damped structures

\section{Introduction}

The literature on lossless positive-real systems is very rich and, as regards the control aspects, is rooted in the papers of Opdenacker and Johnckeere [1,2]. The main concepts related to lossless positive-real systems [3] derive from the classical electric network theory [4,5], where LC circuits are modeled by lossless positive-real systems. Roughly speaking lossless positive-real systems are those systems where, for every trajectory, the energy is exactly equal to that furnished to the system.

Lossless models are widely adopted in several areas of engineering, including electronic, civil, antennas and aerospace engineering. We mention here the design of electrical filters, in particular with the current possibility of realizing inductors in integrated silicon electronic devices [6-8] which makes possible to realize classical LC filters in VLSI technology. Moreover, where large-space flexible structures are dealt with, such as as antennas or solar panels, the need for LQG

Arturo Buscarino

arturo.buscarino@unict.it

1 Department of Electrical, Electronic and Computer Engineering, University of Catania, Viale A. Doria 6, 95125 Catania, Italy

2 CNR-IASI, Italian National Research Council-Institute for Systems Analysis and Computer Science, A.Ruberti, Rome, Italy control techniques is evident [9]. Under this perspective, the approximation of weakly-damped mechanical structures by using lossless positive-real systems has been explored in [2] and the model order reduction of large-scale mechanical systems modeled as lossless positive-real systems by using LQG balancing has been reported in [4].

In [4] interesting results related to continuous-time LQG regulators for passive systems have been discussed, even if the aspects referred to the lossless positive-real systems are not considered. Furthermore, the topic of LQG control of discrete-time lossless positive-real systems [10] has not been addressed in the previous studies. Moreover, several contributions are dedicated to the LQG control of dissipative systems but not to the LQG control of lossless positive-real systems.

Recent papers have been proposed in the area of lossless positive-real systems. In particular in [11] it is proved a numerically stable procedure to derive storage function for linear time-invariant systems. A control theoretical elegant formulation of some classical problems in statistical mechanics that take into account the lossless approximation concept has been introduced in [12].

In the characterization of dissipative systems [13] separating high-loss and lossless components leads to an analytical approach for the study of Lagrangian systems.

In various contributions the relationship between hyperstability theory [14] and the control of lossless positive-real 
systems in the continuous-time case has been dealt with [2] leading to important results. Moreover the case of LQG problem has been discussed in [15].

In this communication, we focus on the determination of straightforward formulas to solve the LQG problem for lossless systems with the aim of obtaining an approximated yet robust solution for weakly-damped systems, thus reducing the numerical complexity of the LQG problem solution.

The following new results are discussed. For the continuous-time case, the expression of the optimal observer gain will be introduced yielding a simple expression of the LQG optimal compensator that proves to be passive [2]. These results (both those referred to the regulator gain and the observer gain) are then demonstrated also for the discretetime case. However, in this case it is shown that, at variance of what is found in continuous-time systems, the compensator is not passive. It is also proved that the LQG compensator is asymptotically stable, therefore it can be passified [16]. This guarantees structural robustness of the closed-loop system, thanks to the hyperstability theory, in spite of some degradation of the optimal performance.

The paper is organized as follows. In Sect. 2 the mathematical preliminaries are presented. In Sect. 3 the main results are discussed. A further section (Sect. 4) regarding numerical examples is included and Sect. 5 concludes the paper, highlighting the main implications of the results discussed in the paper.

\section{Mathematical preliminaries}

Let us consider a linear time-invariant continuous-time system in minimal form:

$\dot{\mathrm{x}}=\mathrm{Ax}+\mathrm{Bu}$

$\mathrm{y}=\mathrm{Cx}+\mathrm{Du}$

where $\mathrm{x} \in \mathbb{R}^{n}, \mathrm{u} \in \mathbb{R}^{m}, \mathrm{y} \in \mathbb{R}^{p}$ with $m=p$.

Definition 1 [17] The realization (1) is said to be externally passive if

$\int_{0}^{t} \mathrm{u}^{T} \mathrm{y} d t \geq 0 \forall t \geq 0$

Definition 2 [18] The transfer function matrix $\mathrm{G}(s)=$ $\mathrm{C}(s \mathrm{I}-\mathrm{A})^{-1} \mathrm{~B}+\mathrm{D}$ is positive-real if the following conditions occur:

- $\mathrm{G}(s)$ is real $\forall s \in \mathbb{R}$;

- $\mathrm{G}(s)+\mathrm{G}^{T}\left(s^{*}\right) \geq 0$;

- any $s$ with $\Re(s) \geq 0$ is not a pole of $\mathrm{G}(s)$.
Definition 3 [18] The lossless positive-real (LPR) systems are a particular class of passive systems for which to every input $\mathrm{u}_{1}(t)$ there corresponds an input $\mathrm{u}_{2}(t)$ for which

$\int_{-\infty}^{\infty} \mathrm{u}^{T}(\tau) \mathrm{y}(\tau) d \tau=0$

where

$\mathrm{u}(t)=\left\{\begin{array}{l}\mathrm{u}_{1}(t) \text { for } t \leq 0 \\ \mathrm{u}_{2}(t) \text { for } t>0\end{array}\right.$

Remark 1 If (1) is a realization of a LPR system, the corresponding transfer function matrix is positive-real with $\mathrm{G}(j \omega)+\mathrm{G}^{T}(-j \omega)=0 \forall \omega \in \mathbb{R}^{+}[18]$.

Another characterization of LPR systems in the time domain is expressed by the following theorem.

Theorem 1 [2] Given a minimal realization (A, B, C, D), it represents a LPR system if there exists a unique matrix $\mathrm{Y} \geq 0$ such that the Kalman-Yakubovitch lemma is satisfied:

$\mathrm{A}^{T} \mathrm{Y}+\mathrm{YA}=0$

$\mathrm{C}^{T}=\mathrm{YB}$

$\mathrm{D}+\mathrm{D}^{T}=0$

Let us now consider a linear time-invariant discrete-time system in minimal form:

$\mathrm{x}(k+1)=\mathrm{Ax}(k)+\mathrm{Bu}(k)$

$\mathrm{y}(k)=\mathrm{Cx}(k)+\mathrm{Du}(k)$

where $\mathrm{x} \in \mathbb{R}^{n}, \mathrm{u} \in \mathbb{R}^{m}, \mathrm{y} \in \mathbb{R}^{p}$ with $m=p$.

Definition 4 [19] The transfer function matrix $\mathrm{G}(z)=$ $\mathrm{C}(z \mathrm{I}-\mathrm{A})^{-1} \mathrm{~B}+\mathrm{D}$ is positive-real if the following conditions occur:

- $\mathrm{G}(z)$ is real $\forall z \in \mathbb{R}$;

- $\mathrm{G}(z)+\mathrm{G}^{T}\left(z^{*}\right) \geq 0$ in $|z|>1$;

- any $z$ with $|z|>1$ is not a pole of $\mathrm{G}(z)$.

Remark 2 If (6) is a realization of LPR system, the corresponding transfer function matrix is positive-real with $\mathrm{G}\left(e^{j \theta}\right)+\mathrm{G}^{T}\left(e^{-j \theta}\right)=0, \forall \omega \in \mathbb{R}^{+}$.

Analogously to Theorem 1, a discrete-time LPR system can be characterized in the time domain.

Theorem 2 [20] Given a minimal realization (A, B, C, D) of a discrete-time system, it represents a LPR system if there exists a unique matrix $\mathrm{Y} \geq 0$ such that:

$$
\begin{aligned}
& \mathrm{Y}-\mathrm{A}^{T} \mathrm{YA}=0 \\
& \mathrm{C}^{T}=\mathrm{A}^{T} \mathrm{YB} \\
& \mathrm{D}+\mathrm{D}^{T}=\mathrm{B}^{T} \mathrm{YB}
\end{aligned}
$$


Let us consider now the Linear Quadratic Gaussian (LQG) control problem. It consist in the design of a normalized linear quadratic regulator that minimizes the quadratic index of the general form

$J=\int_{0}^{\infty}\left(\mathrm{x}^{T} \mathrm{Qx}+\mathrm{u}^{T} \mathrm{Ru}\right) d t$

where $\mathrm{Q}=\mathrm{C}^{T} \mathrm{C}$ and $\mathrm{R}=\mathrm{I}+\mathrm{D}^{T} \mathrm{D}$ are weight matrices, and a Kalman filter with measurement noise with covariance matrix $\mathrm{M}_{v}=\mathrm{I}+\mathrm{DD}^{T}$ and disturbance with covariance matrix $\mathrm{M}_{d}=\mathrm{BB}^{T}$ [21].

The LQG control problem is solved calculating the optimal gain vectors of the linear quadratic regulator $\mathrm{K}_{c}$ and of the Kalman filter $\mathrm{K}_{e}$. This is done by solving the Control Algebraic Riccati Equation (CARE) [21]:

$$
\begin{aligned}
\mathrm{A}^{T} \mathrm{P} & +\mathrm{PA}-\left(\mathrm{PB}+\mathrm{C}^{T} \mathrm{D}\right)\left(\mathrm{I}+\mathrm{D}^{T} \mathrm{D}\right)^{-1}\left(\mathrm{~B}^{T} \mathrm{P}+\mathrm{D}^{T} \mathrm{C}\right) \\
& +\mathrm{C}^{T} \mathrm{C}=0
\end{aligned}
$$

and the dual Filtering Algebraic Riccati Equation (FARE) [21]:

$$
\begin{gathered}
\mathrm{A} \Pi+\Pi \mathrm{A}^{T}-\left(\Pi \mathrm{C}^{T}+\mathrm{BD}^{T}\right)\left(\mathrm{I}+\mathrm{DD}^{T}\right)^{-1} \\
\left(\mathrm{C} \Pi+\mathrm{DB}^{T}\right)+\mathrm{BB}^{T}=0
\end{gathered}
$$

The CARE and FARE admit a unique positive definite solution if the system is in minimal form, so that the associated optimal control and optimal observer gain matrices are

$\mathrm{K}_{c}=\left(\mathrm{I}+\mathrm{D}^{T} \mathrm{D}\right)^{-1}\left(\mathrm{~B}^{T} \overline{\mathrm{P}}+\mathrm{D}^{T} \mathrm{C}\right)$

$\mathrm{K}_{e}=\left(\bar{\Pi} \mathrm{C}^{T}+\mathrm{BD}^{T}\right)\left(\mathrm{I}+\mathrm{DD}^{T}\right)^{-1}$

where $\overline{\mathrm{P}}$ and $\bar{\Pi}$ are the positive definite solutions of the two Riccati Equations (9) and (10) [21].

Proposition 1 The transfer function matrix of the LQG compensator for a continuous-time system is given by

$\mathrm{H}(s)=\mathrm{K}_{c}\left(s \mathrm{I}-\mathrm{A}+\mathrm{BK}_{c}+\mathrm{K}_{e} \mathrm{C}-\mathrm{K}_{e} \mathrm{DK}_{c}\right) \mathrm{K}_{e}$

Let us consider now the discrete-time LQG normalized compensator. The D-CARE equation with $\mathrm{R}=\left(\mathrm{I}+\mathrm{D}^{T} \mathrm{D}\right)$ is [22]

$$
\begin{gathered}
\mathrm{A}^{T} \mathrm{PA}-\mathrm{P}-\left(\mathrm{A}^{T} \mathrm{~PB}+\mathrm{C}^{T} \mathrm{D}\right)\left(\mathrm{B}^{T} \mathrm{~PB}+\mathrm{R}\right)^{-1} \\
\left(\mathrm{~B}^{T} \mathrm{PA}+\mathrm{D}^{T} \mathrm{C}\right)+\mathrm{C}^{T} \mathrm{C}=0
\end{gathered}
$$

The normalized D-FARE equation [22] with $\mathrm{R}^{*}=$ $\left(\mathrm{I}+\mathrm{DD}^{T}\right)$ is

$$
\begin{gathered}
\mathrm{A}^{T} \Pi \mathrm{A}-\Pi-\left(\mathrm{A}^{T} \Pi \mathrm{B}+\mathrm{C}^{T} \mathrm{D}\right)\left({\left.\mathrm{C} \Pi \mathrm{C}^{T}+\mathrm{R}^{*}\right)^{-1}}^{\left(\mathrm{C}^{2} \mathrm{~A}^{T}+\mathrm{D}^{T} \mathrm{~B}^{T}\right)+\mathrm{BB}^{T}=0}\right.
\end{gathered}
$$

The D-CARE and D-FARE admit a unique positive definite solution if the system is in minimal form, from which the optimal control and optimal observer gain can be determined [22] as:

$$
\begin{aligned}
& \mathrm{K}_{c}=\left(\mathrm{B}^{T} \overline{\mathrm{P}} \mathrm{B}+\mathrm{R}\right)^{-1}\left(\mathrm{~B}^{T} \overline{\mathrm{P}} \mathrm{A}+\mathrm{D}^{T} \mathrm{C}\right) \\
& \mathrm{K}_{e}=\left(\mathrm{A} \bar{\Pi} \mathrm{C}^{T}+\mathrm{BD}^{T}\right)\left(\mathrm{C} \bar{\Pi} \mathrm{C}^{T}+\mathrm{R}^{*}\right)^{-1}
\end{aligned}
$$

Proposition 2 The transfer function matrix of the $L Q G \mathrm{com}$ pensator of a discrete-time system is given by

$\mathrm{H}(z)=\mathrm{K}_{c}\left(z \mathrm{I}-\mathrm{A}+\mathrm{BK}_{c}+\mathrm{K}_{e} \mathrm{C}-\mathrm{K}_{e} \mathrm{DK}_{c}\right) \mathrm{K}_{e}$

\section{The LQG compensator for lossless positive-real system}

\subsection{The continuous-time case}

Proposition 3 The optimal LQR gain matrix for a LPR system in minimal form is given by

$\mathrm{K}_{c}=(\mathrm{I}+\mathrm{D})^{-1} \mathrm{C}$

Proof The proof is based on the uniqueness of the positive definite solution of the Riccati equation. For this reason, the matrix $\overline{\mathrm{P}}$ in (9) and the matrix $\mathrm{Y}$ in (5) are equal. Let us then consider a state-space realization of a LPR system (A, B, C, D). Substituting in Eq. (9) the definition of $\mathrm{K}_{c}$ as in Eq. (11) and plugging Eqs. (5), it follows that

$-\mathrm{C}^{T}(\mathrm{I}+\mathrm{D}) \mathrm{K}_{c}+\mathrm{C}^{T} \mathrm{C}=0$

From this, one derives that $(\mathrm{I}+\mathrm{D}) \mathrm{K}_{c}=\mathrm{C}$ and, thus, $\mathrm{K}_{c}=(\mathrm{I}+\mathrm{D})^{-1} \mathrm{C}$.

Proposition 4 The optimal observer gain matrix for a LPR system in minimal form is given by

$\mathrm{K}_{e}=\mathrm{B}\left(\mathrm{I}+\mathrm{D}^{T}\right)^{-1}$

Proof The proof follows the same steps in the proof of Prop. 3. Let us consider a state-space realization of the LPR system (A, B, C, D) and include the relation in Eqs. (5) 
within the FARE in Eq. (10), after substituting in it the gain as defined in Eq. (11). It follows that

$$
-\mathrm{K}_{e}\left(\mathrm{I}+\mathrm{D}^{T}\right) \mathrm{B}^{T}+\mathrm{BB}^{T}=0
$$

It is possible to derive that $\mathrm{K}_{e}\left(\mathrm{I}+\mathrm{D}^{T}\right)=\mathrm{B}$, from which it follows $\mathrm{K}_{e}=\mathrm{B}\left(\mathrm{I}+\mathrm{D}^{T}\right)^{-1}$.

Remark 3 The LQG compensator of a continuous-time positive-real system with weight matrices chosen as in (9) and (10) is a strictly positive-real system [23]. Therefore, also the LQG compensator for a continuous-time LPR system with $\mathrm{K}_{c}$ and $\mathrm{K}_{e}$ as in Eqs. (21) and (23) is strictly positive-real.

\subsection{The discrete-time case}

Proposition 5 The optimal LQR gain matrix for a discretetime LPR system in minimal form is given by

$\mathrm{K}_{c}=(\mathrm{I}+\mathrm{D})^{-1} \mathrm{C}$

Proof Also for discrete-time systems, there is a unique positive definite solution of the D-CARE. This implies that the matrix $\overline{\mathrm{P}}$ in (13) and the matrix $\mathrm{Y}$ in (7) are the same. Let us consider, then, a state-space representation of a discretetime LPR system (A, B, C, D). Substituting Eqs. (7) in the D-CARE (13), after including the relationship for $\mathrm{K}_{c}$ in (15), it follows that

$-\mathrm{C}^{T}(\mathrm{I}+\mathrm{D}) \mathrm{K}_{c}+\mathrm{C}^{T} \mathrm{C}=0$

From this, it is derived that $(\mathrm{I}+\mathrm{D}) \mathrm{K}_{c}=\mathrm{C}$ from which $\mathrm{K}_{c}=(\mathrm{I}+\mathrm{D})^{-1} \mathrm{C}$.

Proposition 6 The optimal observer gain matrix for a discrete time LPR system in minimal form is given as

$\mathrm{K}_{e}=\mathrm{B}\left(\mathrm{I}+\mathrm{D}^{T}\right)^{-1}$

Proof Consider a state-space realization of the discrete-time LPR system (A, B, C, D), and plug Eqs. (7) in (14) including the relationship in (15) for the $\mathrm{K}_{e}$ to find:

$-\mathrm{K}_{e}\left(\mathrm{I}+\mathrm{D}^{T}\right) \mathrm{B}^{T}+\mathrm{BB}^{T}=0$

This yields to $\mathrm{K}_{e}\left(\mathrm{I}+\mathrm{D}^{T}\right)=\mathrm{B}$ and so Eq. (23) follows.

Interestingly, the property of positive-realness of the LQG compensator discussed in Remark 3 for continuous-time systems in general is not true in the discrete-time case. In fact, even if the design structure in both the continuous and discrete-time case is equal, we notice that both are strictly proper systems. Taking into account the discrete-time positive-real lemma [20] there must exist a positive definite matrix $\overline{\mathrm{P}}$ such that

$\overline{\mathrm{P}}-\mathrm{A}_{C}^{T} \overline{\mathrm{P}} \mathrm{A}_{C}=\mathrm{L}^{T} \mathrm{~L}$

$\mathrm{C}^{T}-\mathrm{A}_{C}^{T} \overline{\mathrm{P}} \mathrm{B}=\mathrm{L}^{T} \mathrm{~W}$

$\mathrm{D}_{C}+\mathrm{D}_{C}^{T}-\mathrm{B}^{T} \overline{\mathrm{P}} \mathrm{B}=\mathrm{W}^{T} \mathrm{~W}$

being $\left(\mathrm{A}_{C}, \mathrm{~B}_{C}, \mathrm{C}_{C}\right.$ and $\left.\mathrm{D}_{C}\right)$ a state-space representation of the compensator transfer function matrix. Since $\mathrm{D}_{C}=0$, the third equation in (25) cannot be satisfied and hence the system is not passive.

The following result, however, ensures the stability of the LQG optimal compensator for discrete-time LPR systems.

Proposition 7 Given a minimal representation (A, B, C, D) of a discrete-time LPR system, the optimal LQG compensator is asymptotically stable.

Proof The proof is based on the evaluation of the discretetime Lyapunov equation for the state matrix $\mathrm{A}_{C}=\mathrm{A}-$ $\mathrm{BK}_{c}-\mathrm{K}_{e} \mathrm{C}+\mathrm{K}_{e} \mathrm{DK}_{c}$ of the optimal compensator, choosing as positive definite solution the matrix $\mathrm{P}$ satisfying (7). It yields

$$
\begin{aligned}
\mathrm{A}_{C}= & \mathrm{A}-\mathrm{B}(\mathrm{I}+\mathrm{D})^{-1} \mathrm{C}-\mathrm{B}\left(\mathrm{I}+\mathrm{D}^{T}\right)^{-1} \\
& \mathrm{C}+\mathrm{B}\left(\mathrm{I}+\mathrm{D}^{T}\right)^{-1} \mathrm{D}(\mathrm{I}+\mathrm{D})^{-1} \mathrm{C}= \\
= & \mathrm{A}-\mathrm{B}\left[\left(\mathrm{I}+\mathrm{D}^{T}\right)^{-1}+\mathrm{I}\right](\mathrm{I}+\mathrm{D})^{-1} \mathrm{C}
\end{aligned}
$$

and therefore, defining $\mathrm{M}=\left[\left(\mathrm{I}+\mathrm{D}^{T}\right)^{-1}+\mathrm{I}\right](\mathrm{I}+\mathrm{D})^{-1}$, we get

$$
\begin{aligned}
& (\mathrm{A}-\mathrm{BMC})^{T} \mathrm{P}(\mathrm{A}-\mathrm{BMC})-\mathrm{P} \\
& =\mathrm{A}^{T} \mathrm{PA}-\mathrm{A}^{T} \mathrm{PBMC}-\mathrm{C}^{T} \mathrm{M}^{T} \mathrm{~B}^{T} \mathrm{PA} \\
& \quad+\mathrm{C}^{T} \mathrm{M}^{T} \mathrm{~B}^{T} \mathrm{PBMC} \\
& =-\mathrm{C}^{T}\left(\mathrm{M}+\mathrm{M}^{T}-\mathrm{M}^{T}\left(\mathrm{D}+\mathrm{D}^{T}\right) \mathrm{M}\right) \mathrm{C}
\end{aligned}
$$

In order to prove the thesis, we need to prove that the matrix

$$
\left(\mathrm{M}+\mathrm{M}^{T}-\mathrm{M}^{T}\left(\mathrm{D}+\mathrm{D}^{T}\right) \mathrm{M}\right)
$$

is positive definite. 
To show this, consider that

$$
\begin{aligned}
\mathrm{M}+ & \mathrm{M}^{T}-\mathrm{M}^{T}\left(\mathrm{D}+\mathrm{D}^{T}\right) \mathrm{M} \\
= & {\left[\left(\mathrm{I}+\mathrm{D}^{T}\right)^{-1}+\mathrm{I}\right](\mathrm{I}+\mathrm{D})^{-1} } \\
& +\left(\mathrm{I}+\mathrm{D}^{T}\right)^{-1}\left[(\mathrm{I}+\mathrm{D})^{-1}+\mathrm{I}\right] \\
& -\left(\mathrm{I}+\mathrm{D}^{T}\right)^{-1}\left[(\mathrm{I}+\mathrm{D})^{-1}+\mathrm{I}\right]\left(\mathrm{D}+\mathrm{D}^{T}\right) \\
& \times\left[\left(\mathrm{I}+\mathrm{D}^{T}\right)^{-1}+\mathrm{I}\right](\mathrm{I}+\mathrm{D})^{-1} \\
= & \left(\mathrm{I}+\mathrm{D}^{T}\right)^{-1}\left[2 \mathrm{I}+\mathrm{D}^{T}\right](\mathrm{I}+\mathrm{D})^{-1} \\
& +\left(\mathrm{I}+\mathrm{D}^{T}\right)^{-1}[2 \mathrm{I}+\mathrm{D}](\mathrm{I}+\mathrm{D})^{-1} \\
& -\left(\mathrm{I}+\mathrm{D}^{T}\right)^{-1}\left[\mathrm{D}+\mathrm{D}^{T}+\left(\mathrm{D}+\mathrm{D}^{T}\right)\left(\mathrm{I}+\mathrm{D}^{T}\right)^{-1}\right. \\
& +\left(\mathrm{I}+\mathrm{D}^{-1}\left(\mathrm{D}+\mathrm{D}^{T}\right)\right. \\
& \left.+(\mathrm{I}+\mathrm{D})^{-1}\left(\mathrm{D}+\mathrm{D}^{T}\right)\left(\mathrm{I}+\mathrm{D}^{T}\right)^{-1}\right](\mathrm{I}+\mathrm{D})^{-1} \\
= & \left(\mathrm{I}+\mathrm{D}^{T}\right)^{-1}\left\{(\mathrm{I}+\mathrm{D})^{-1}\right. \\
& \times\left[4 \mathrm{I}+\mathrm{D}^{-1} \mathrm{D}^{T}+2 \mathrm{DD} \mathrm{D}^{T}-\mathrm{D}^{2}-\mathrm{D}^{T^{2}}\right] \\
& \left.\times\left(\mathrm{I}+\mathrm{D}^{T}\right)^{-1}\right\}(\mathrm{I}+\mathrm{D})^{-1}
\end{aligned}
$$

After some matrix manipulations, we obtain

$$
\begin{aligned}
& \mathrm{M}+\mathrm{M}^{T}-\mathrm{M}^{T}\left(\mathrm{D}+\mathrm{D}^{T}\right) \mathrm{M} \\
& \quad=\left(\mathrm{I}+\mathrm{D}^{T}\right)^{-1}\left\{(\mathrm{I}+\mathrm{D})^{-1}\right. \\
& \left.\left[2 \mathrm{I}+2(\mathrm{I}+\mathrm{D})^{-1}\left(\mathrm{I}+\mathrm{D}^{T}\right)^{-1}\right]\left(\mathrm{I}+\mathrm{D}^{T}\right)^{-1}\right\}(\mathrm{I}+\mathrm{D})^{-1}
\end{aligned}
$$

that is a positive definite matrix, from which the thesis follows.

\section{Numerical results}

Example 1 Let us consider the LPR continuous-time system

$$
G(s)=\frac{1}{s}
$$

Given $(A, B, C, D)$ a state-space representation, with $A=0$, $B=1, C=1, D=0$, the optimal compensator can be calculated as

$$
\begin{aligned}
H(s)= & K_{c}\left(s-A+B K_{c}+K_{e} C-K_{e} D K_{c}\right) K_{e} \\
= & (1+D)^{-1} C\left(s-A+B(1+D)^{-1} C\right. \\
& +B(1+D)^{-1} C \\
& \left.-B(1+D)^{-1} D(1+D)^{-1} C\right) B(1+D)^{-1}= \\
= & \frac{1}{s+2}
\end{aligned}
$$

which is a positive-real system.

Example 2 Let us now consider the LPR discrete-time system

$$
G(z)=\frac{z+1}{z-1}
$$

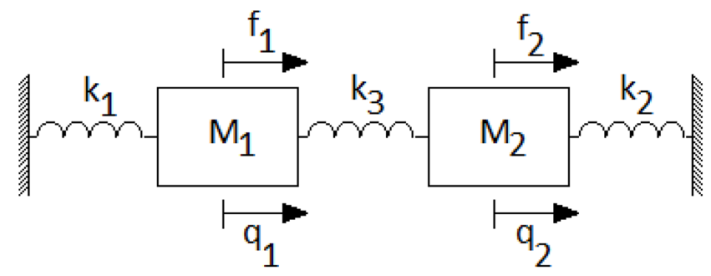

Fig. 1 Undamped flexible structure

which admits a state-space representation $(A, B, C, D)$ with $A=1, B=1, C=2, D=1$. The optimal compensator can be obtained as:

$$
\begin{aligned}
& H(z)=K_{c}\left(z-A+B K_{c}+K_{e} C-K_{e} D K_{c}\right) K_{e} \\
& =(1+D)^{-1} C\left(z-A+B(1+D)^{-1} C+B(1+D)^{-1} C\right. \\
& \left.-B(1+D)^{-1} D(1+D)^{-1} C\right) B(1+D)^{-1} \\
& =\frac{1}{2} \frac{1}{z+0.5}
\end{aligned}
$$

which is stable but not positive-real.

Example 3 Let us consider the mechanical system reported in Fig. 1. It represents a mass-spring system subjected to the two forces $f_{1}$ and $f_{2}$, which are the inputs of the system. Choosing as outputs the velocity of the two masses $m_{1}$ and $m_{2}$ and defining as state vector $\mathrm{x}=\left[\begin{array}{llll}q_{1} & q_{2} & \dot{q}_{1} & \dot{q}_{2}\end{array}\right]^{T}$ the system is LPR and admits the folling state-space representation:

$$
\mathrm{A}=\left[\begin{array}{cc}
0 & \mathrm{I} \\
-\mathrm{M}^{-1} \mathrm{~K} & 0
\end{array}\right] ; \mathrm{B}=\left[\begin{array}{c}
0 \\
\mathrm{M}^{-1}
\end{array}\right] ; \mathrm{C}=\left[\begin{array}{ll}
0 & \mathrm{I}
\end{array}\right]
$$

where $\mathrm{M}=\left[\begin{array}{cc}m_{1} & 0 \\ 0 & m_{2}\end{array}\right]$ and $\mathrm{K}=\left[\begin{array}{cc}k_{1}+k_{3} & -k_{3} \\ -k_{3} & k_{2}+k_{3}\end{array}\right]$, with $k_{1}, k_{2}$ and $k_{3}$ the springs constants, and I the $2 \times 2$ identity matrix.

Consider for instance the following values of the parameters $m_{1}=6, m_{2}=0.4, k_{1}=1, k_{2}=2$, and $k_{3}=0.3$, we get the following state space representation:

$$
\mathrm{A}=\left[\begin{array}{cccc}
0 & 0 & 1 & 0 \\
0 & 0 & 0 & 1 \\
-\frac{13}{60} & 0.05 & 0 & 0 \\
0.75 & -5.75 & 0 & 0
\end{array}\right] ; \mathrm{B}=\left[\begin{array}{cc}
0 & 0 \\
0 & 0 \\
\frac{1}{6} & 0 \\
0 & 2.5
\end{array}\right] ; \mathrm{C}=\left[\begin{array}{llll}
0 & 0 & 1 & 0 \\
0 & 0 & 0 & 1
\end{array}\right]
$$

The optimal LQG compensator can be calculated as:

$$
\begin{aligned}
& \mathrm{H}(s)=\mathrm{K}_{c}\left(s \mathrm{I}-\mathrm{A}+\mathrm{BK}_{c}+\mathrm{K}_{e} \mathrm{C}-\mathrm{K}_{e} \mathrm{DK}_{c}\right) \mathrm{K}_{e} \\
& =\left[\begin{array}{ll}
H_{11}(s) & H_{12}(s) \\
H_{21}(s) & H_{22}(s)
\end{array}\right]
\end{aligned}
$$




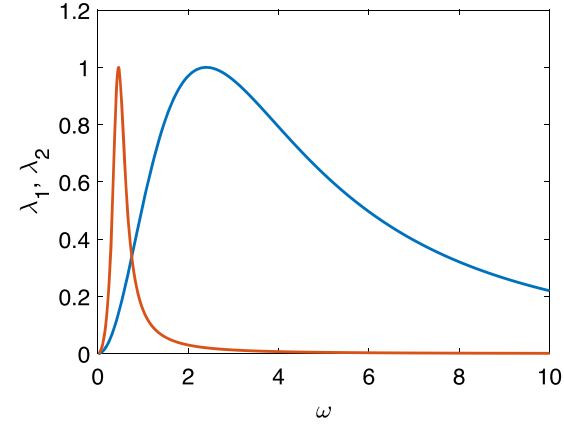

Fig. 2 Spectrum of matrix $\overline{\mathrm{H}}=\mathrm{H}(j \omega)+\mathrm{H}^{T}(-j \omega)$

with

$H_{11}(s)=\frac{0.1667 s^{3}+0.8333 s^{2}+0.9583 s}{s^{4}+5.333 s^{3}+7.633 s^{2}+3 s+1.208}$

$H_{12}(s)=H_{21}(s)=\frac{0.125 s}{s^{4}+5.333 s^{3}+7.633 s^{2}+3 s+1.208}$

and

$H_{22}(s)=\frac{2.5 s^{3}+0.8333 s^{2}+0.5417 s}{s^{4}+5.333 s^{3}+7.633 s^{2}+3 s+1.208}$

Let us consider now the spectrum of the matrix $\overline{\mathrm{H}}=$ $\mathrm{H}(j \omega)+\mathrm{H}^{T}(-j \omega)$. According to Definition 2, in order to be $\mathrm{H}(s)$ a passive compensator, $\overline{\mathrm{H}}$ must be a positive definite matrix. In Fig. 2, the eigenvalues $\lambda_{1}$ and $\lambda_{2}$ of $\bar{H}$ are reported as a function of $\omega$ and are positive $\forall \omega$, since they are positive for any $\omega$, we find that the LQG optimal compensator of the lossless positive-real MIMO system is passive.

Example 4 Let us consider the discrete-time LPR system characterized by the following state-space representation:

$$
\begin{aligned}
& \mathrm{A}=\left[\begin{array}{cccc}
0.6514 & 0.0122 & 1.6514 & 0.0122 \\
0.1835 & -0.7023 & 0.1835 & 0.2977 \\
-0.3486 & 0.0122 & 0.6514 & 0.0122 \\
0.1835 & -1.7023 & 0.1835 & -0.70230
\end{array}\right] \\
& \mathrm{B}=\left[\begin{array}{ll}
0.2752 & 0.0306 \\
0.0306 & 0.7441 \\
0.2752 & 0.0306 \\
0.0306 & 0.74410
\end{array}\right] \\
& C=\left[\begin{array}{cccc}
-0.1743 & 0.0061 & 0.8257 & 0.0061 \\
0.0917 & -0.8512 & 0.0917 & 0.1488
\end{array}\right] \\
& \mathrm{D}=\left[\begin{array}{ll}
0.1376 & 0.0153 \\
0.0153 & 0.3721
\end{array}\right]
\end{aligned}
$$

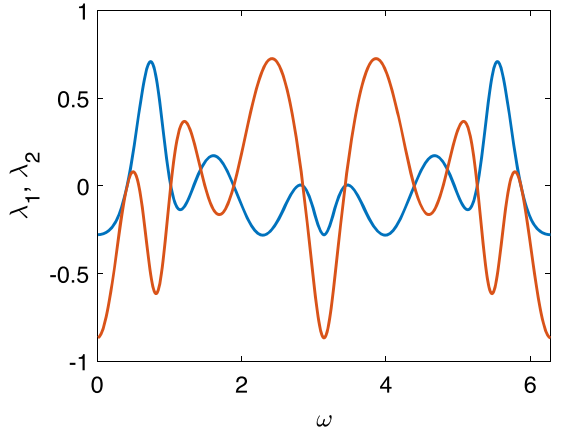

Fig. 3 Spectrum of matrix $\overline{\mathrm{H}}=\mathrm{H}\left(e^{j \omega}\right)+\mathrm{H}^{T}\left(e^{-j \omega}\right)$

The LQG optimal compensator is calculated as:

$$
\begin{aligned}
\mathrm{H}(z) & =\mathrm{K}_{c}\left(s \mathrm{I}-\mathrm{A}+\mathrm{BK}_{c}+\mathrm{K}_{e} \mathrm{C}-\mathrm{K}_{e} \mathrm{DK}_{c}\right) \mathrm{K}_{e} \\
& =\left[\begin{array}{ll}
H_{11}(z) & H_{12}(z) \\
H_{21}(z) & H_{22}(z)
\end{array}\right]
\end{aligned}
$$

with

$$
\begin{aligned}
& H_{11}(z)=\frac{0.1383 z^{3}-0.1227 z^{2}-0.1482 z+0.01742}{z^{4}-0.3371 z^{3}-0.2054 z^{2}+0.4078 z-0.04507} \\
& \quad H_{12}(z) \\
& \quad=H_{21}(z) \\
& \quad=\frac{0.02075 z^{3}+0.0002172 z^{2}-0.02209 z-0.01448}{z^{4}-0.3371 z^{3}-0.2054 z^{2}+0.4078 z-0.04507}
\end{aligned}
$$

and

$H_{22}(z)=\frac{-0.2751 z^{3}-0.1194 z^{2}+0.2446 z-0.2045}{z^{4}-0.3371 z^{3}-0.2054 z^{2}+0.4078 z-0.04507}$.

Let us evaluate the spectrum of the matrix $\overline{\mathrm{H}}=\mathrm{H}\left(e^{j \omega}\right)+$ $\mathrm{H}^{T}\left(e^{-j \omega}\right)$. According to Definition 4, in order to be $\mathrm{H}(z)$ a passive compensator, $\overline{\mathrm{H}}$ must be a positive definite matrix. The eigenvalues $\lambda_{1}$ and $\lambda_{2}$ of $\overline{\mathrm{H}}$ are reported in Fig. 3 as a function of $\omega$. Since they assume negative values for some $\omega$, the LQG optimal compensator of the lossless positive-real discrete-time MIMO system is not passive as expected.

Example 5 This example allows us to investigate the possibility to get a positive-real LQG optimal compensator for a discrete-time lossless positive-real system. As discussed in Example 3., the compensator obtained therein, i.e. $H(z)=$ $\frac{1}{2} \frac{1}{z+0.5}$, is stable but not positive-real. Following the strategy outlined in [16], it is possible to apply a forward action $\alpha \mathrm{I}$ to system $H(z)$ in order to make it positive-real. The Nyquist plot reported in Fig. 4a allows to graphically determine the minimum value of $\alpha$ needed to make the system positivereal, i.e. $\alpha=1$, as shown in the plot reported in Fig. $4 \mathrm{~b}$ that is related to the system $H(z)+\alpha$. 
Fig. 4 Example 5: design of a positive-real discrete-time LQG optimal compensator. a Nyquist plot of $H(z)$ as in Example 3; b Nyquist plot of $H(z)+\alpha$ with $\alpha=1$

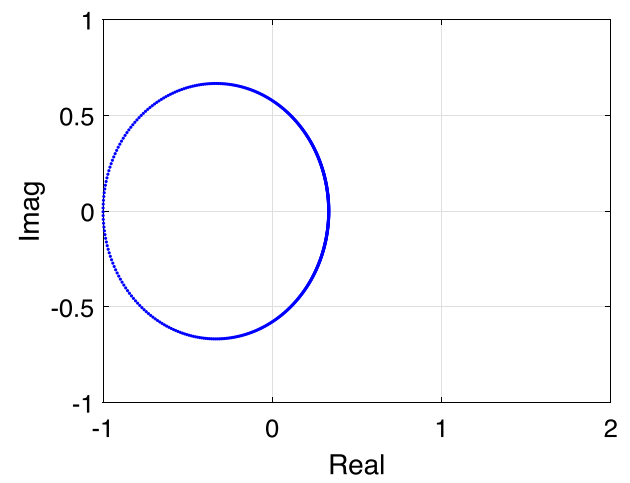

(a)

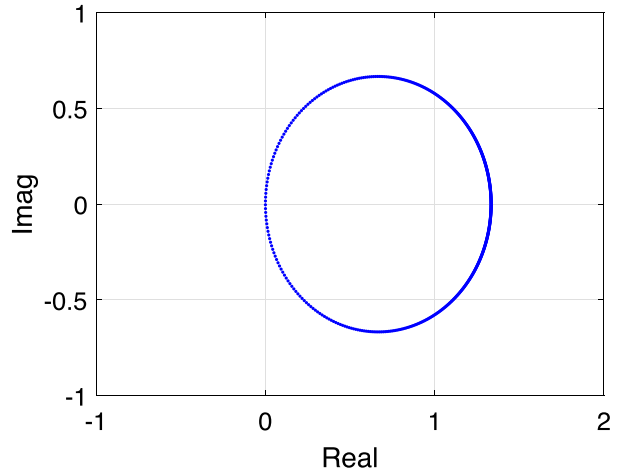

(b)
We remark that including a forward action makes the LQG compensator a strictly proper system, thus in this case the value of $\alpha$ can be analytically derived by solving Eqs. (25) with $D=\alpha$. The condition $D=\alpha>1$ can be retrieved to ensure the existence of a unique positive definite solution $P$. In virtue of the hyperstability, the positive-real compensator obtained with these procedures guarantees the asymptotical stability of the closed-loop system when the plant is stable.

We now consider two examples of weakly-damped systems and study whether it is possible to use as an approximation of the optimal case the gain vector obtained for the corresponding lossless system, i.e., the system derived assuming that the damping is exactly zero. In particular, we will show that using this gain will yield an asymptotically stable closed-loop systems without the need of calculating the actual optimal gain vector solving the LQG optimal control problem, but directly adopting the formulas presented in this paper. We remark that asymptotic stability is ensured by the hyperstability theorem [14] as the the weakly-damped system and the LQG compensator calculated for lossless systems are always positive-real.

Example 6 Let us consider the two transfer functions

$$
\begin{aligned}
& G_{1}(s)=\frac{s}{s^{2}+k_{1} s+2} \\
& G_{2}(s)=\frac{s}{s^{2}+k_{2} s+1}
\end{aligned}
$$

and the system $G(s)$ given by the series of $G_{1}(s)$ and $G_{2}(s)$ as

$$
G(s)=\frac{2 s^{3}+\left(k_{1}+k_{2}\right) s^{2}+3 s}{s^{4}+\left(k_{1}+k_{2}\right) s^{3}+\left(k_{1} k_{2}+3\right) s^{2}+\left(k_{1}+2 k_{2}\right) s+2}
$$

For $k_{1}=k_{2}=0, k_{1}=k_{2}=0, G(s)$ is a lossless positivereal continuous-time system. In this case, given a state-space representation (A, B, C), the LQG optimal gain vector can be determined as $\mathrm{K}_{c}=\mathrm{C}$.

Let us consider now the case $k_{1} \neq 0$ and $k_{2} \neq 0$ and let us define the parameter $\delta_{m}$ as the average Euclidean distance

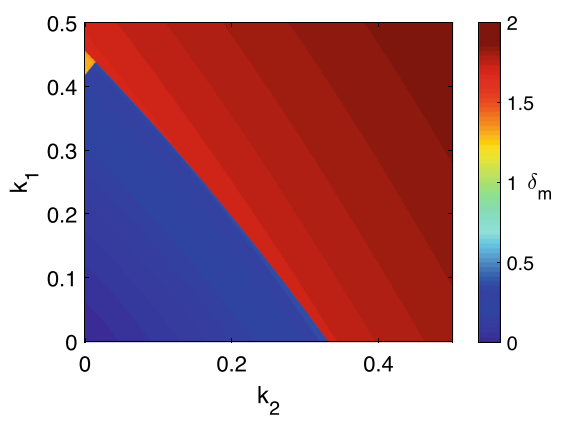

Fig. 5 Example 6: average distance $\delta_{m}$ between the nominal optimal poles $\mathcal{E}$ and the set of poles $\mathcal{E}_{L L}$ obtained for $\mathrm{K}_{c}=\mathrm{C}$ in the space $k_{1}-k_{2}$

between the set of the nominal optimal poles $\mathcal{E}$ and the set of poles $\mathcal{E}_{L L}$ obtained for $\mathrm{K}_{c}=\mathrm{C}$. The map reported in Fig. 5 shows the trend of $\delta_{m}$ varying $k_{1}$ and $k_{2}$. The location of the poles $\mathcal{E}_{L L}$ is actually near to the optimal poles $\mathcal{E}$ of the damped system, thus ensuring a good approximation even for increasing values of the damping factors. It is interesting to note that the trend of $\delta_{m}$ undergoes a sharp transition which corresponds to a pair of complex poles collapsing on the real axis. This behavior is also evident if we move along the diagonal of Fig. 5. Consider the case $k_{1}=k_{2}=k$ and let $\mathrm{k}$ vary by in the range $[0,1]$. In Fig. 6a the two sets $\mathcal{E}$ (red circles) and $\mathcal{E}_{L L}$ (blue circles) are reported. The corresponding trend of $\delta_{m}$ is shown in Fig. 6b, where the sharp transition is clearly observed at $k=0.2$ for which we have two coincident real poles.

Example 7 In order to verify the generality of the numerical results obtained in Example. 5, let us know consider the model of the tetrahedral truss of the Draper Laboratory [24]. It consist in $6 \times 6$ transfer function matrix populated by blocks of the form

$G_{i j}(s)=\sum_{h=1}^{12} \frac{s}{s^{2}+2 \xi \omega_{h} s+\omega_{h^{2}}}$

with $i=1, \ldots, 6$ and $j=1, \ldots, 6$ and where the values of $\omega_{h}$ are reported in Table 1 and $\xi$ accounts for the structure 
Fig. 6 Example 6: comparison of the nominal optimal poles $\mathcal{E}$ and the set of poles $\mathcal{E}_{L L}$ obtained for $\mathrm{K}_{c}=\mathrm{C}$ for $k_{1}=k_{2}=k$. a Location of the poles $\mathcal{E}$ (red circles) and the set of poles $\mathcal{E}_{L L}$ (blue circles); $\mathbf{b}$ trend of the corresponding $\delta_{m}$. (Color figure online)

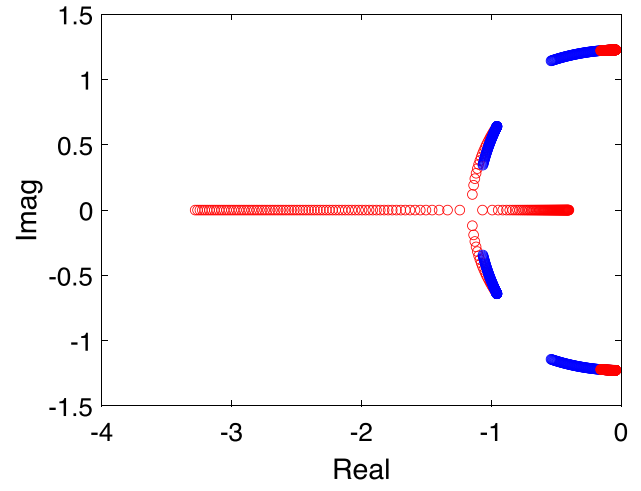

(a)

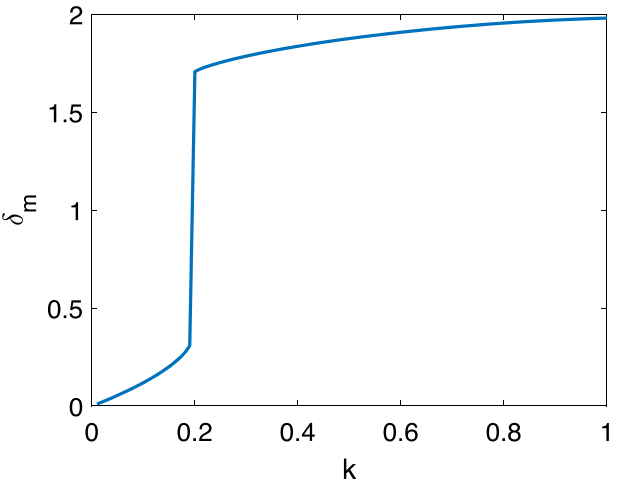

(b)
Table 1 Natural frequencies of the model for the tetrahedral truss of the Draper Laboratory [2]

\begin{tabular}{ll}
\hline$\omega_{1}$ & $1.34 \mathrm{rad} / \mathrm{s}$ \\
$\omega_{2}$ & $1.66 \mathrm{rad} / \mathrm{s}$ \\
$\omega_{3}$ & $2.89 \mathrm{rad} / \mathrm{s}$ \\
$\omega_{4}$ & $2.95 \mathrm{rad} / \mathrm{s}$ \\
$\omega_{5}$ & $3.39 \mathrm{rad} / \mathrm{s}$ \\
$\omega_{6}$ & $4.20 \mathrm{rad} / \mathrm{s}$ \\
$\omega_{7}$ & $4.66 \mathrm{rad} / \mathrm{s}$ \\
$\omega_{8}$ & $4.76 \mathrm{rad} / \mathrm{s}$ \\
$\omega_{9}$ & $8.35 \mathrm{rad} / \mathrm{s}$ \\
$\omega_{10}$ & $9.25 \mathrm{rad} / \mathrm{s}$ \\
$\omega_{11}$ & $10.28 \mathrm{rad} / \mathrm{s}$ \\
$\omega_{12}$ & $12.91 \mathrm{rad} / \mathrm{s}$ \\
\hline
\end{tabular}

damping. It can be verified that the model is lossless when $\xi=0$.

Let us now evaluate $\delta_{m}$ increasing the value of $\xi$ in the range $[0,1]$. The error introduced by considering the optimal gain of the lossless approximation (blue curve in Fig. 7a) increases linearly with a sharper slope for values of $\xi<0.01$ and then the slope is reduced and maintained almost constant up to $\xi=1$, thus confirming the validity of considering as optimal gain $\mathrm{K}_{c}=\mathrm{C}$ as for the lossless approximation. The poles locus reported in Fig. 7b clearly shows that the error is mainly due to the high frequency real pole.

Suppose now to consider parameters affected by uncertainty, i.e. the damping factors of the 12 modes are $\xi_{k}=\xi \rho_{k}$, where $\rho$ is a stochastic variable drawn from a uniform distribution in the range $[0,1]$, and let us calculate $\delta_{m}$ for $\xi \in[0,1]$. We obtain the curve reported in red in Fig. 7a, averaged over 100 realizations. Again the use of the optimal gain for the lossless approximation ensures an effective control.

\section{Conclusions}

The lossless systems assume a fundamental importance in various areas of engineering as, in many of these cases, a suitable lossless approximation of real weakly-damped largescale flexible structures strongly simplifies the numerical procedure to solve the LQG problem. Complex engineering structures with low damping properties as deep space network antennas and solar panels for satellites power supply are classical engineering examples in which an active compensation based on the LQG problem can be formulated by considering lossless approximate models.

In this paper, the structure of the LQG compensator for LPR continuous-time and discrete-time systems has been discussed. Specific relationships to determine the LQG gain vectors have been introduced leading to the result that the compensator cannot be passive in the discrete-time case.

The paper introduces an immediate procedure to obtain the optimal gains, thus avoiding to solve the two Riccati equations. The direct use of the introduced formulas has been proved to be effective in the design of LQG optimal control of real low-damping flexible structures with the advantage of an immediate design procedure that in any case guarantees the closed-loop stability of the system since the feedback configurations consists of a passive system and a passive compensator [14]. Indeed the numerical analysis carried out shows that the performance decrease is marginal as the closed-loop poles do not largely deviate from the nominal locations.

The same considerations are also valid for the discretetime case. Moreover, for them a forward action in order to make positive-real the compensator must be used in synergy with the feedback configuration. A trivial error that can be made in the design of the optimal compensator for discrete-time systems, in fact, is to perform the design in the continuous-time domain and then apply a bilinear transformation to obtain the compensator $\mathrm{H}(z)$ : the compensator obtained in this way is not the optimal compensator. We have seen that the compensator for discrete-time LPR systems is, in fact, not passive. However, leveraging the results of [16], it can be made passive by the use of a forward action. Clearly, this deteriorates the performance, yielding a control action that is sub-optimal, but prompts for a passive realization. 
Fig. 7 Example 7: comparison of the nominal optimal poles $\mathcal{E}$ and the set of poles $\mathcal{E}_{L L}$ obtained for $\mathrm{K}_{c}=$ C. a Trend of $\delta_{m}: \xi \in[0,1]$ (blue curve), $\xi_{k}=\xi \rho_{k}$, with $\rho$ drawn from a uniform distribution in the range $[0,1]$ (red curve, averaged over 100 realizations); $\mathbf{b}$ location of the poles $\mathcal{E}$ (red dots) and the set of poles $\mathcal{E}_{L L}$ (blue dots). (Color figure online)

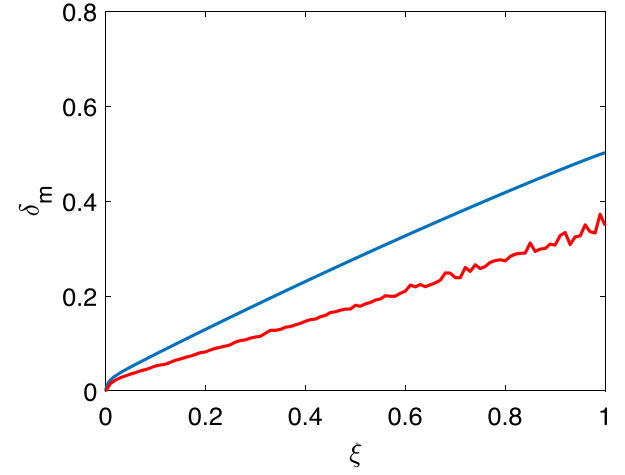

(a)

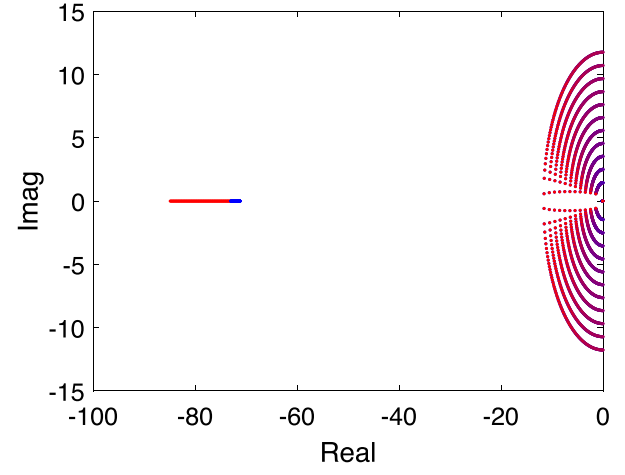

(b)
Funding Open access funding provided by Università degli Studi di Catania within the CRUI-CARE Agreement.

Authors contribution: $\mathrm{AB}$ and $\mathrm{LF}$ developed the main calculations of the paper, MB and MF discussed and analyzed the results. All the Authors wrote the manuscript.

Availability of data and material All relevant data are included in the text.

Open Access This article is licensed under a Creative Commons Attribution 4.0 International License, which permits use, sharing, adaptation, distribution and reproduction in any medium or format, as long as you give appropriate credit to the original author(s) and the source, provide a link to the Creative Commons licence, and indicate if changes were made. The images or other third party material in this article are included in the article's Creative Commons licence, unless indicated otherwise in a credit line to the material. If material is not included in the article's Creative Commons licence and your intended use is not permitted by statutory regulation or exceeds the permitted use, you will need to obtain permission directly from the copyright holder. To view a copy of this licence, visit http://creativecomm ons.org/licenses/by/4.0/.

\section{References}

1. Jonckheere EA, Opdenacker P (1984) Singular value analysis, balancing, and model reduction of large space structures. In: Americal Control Conference, pp 141-149

2. Opdenacker P, Jonckheere EA (1985) LQG balancing and reduced LQG compensation of symmetric passive systems. Int J Control 41:73-109

3. Hakimi-Moghaddam M (2020) Positive real and strictly positive real MIMO systems: theory and application. Int J Dyn Control 8:448-458

4. Jonckheere EA, Opdenacker P (1987) Characterization of passive systems through their closed-loop LQG characteristic values. IEEE Trans Circ Syst 34:324-326

5. Anderson BD, Vongpanitlerd S (2013) Network analysis and synthesis: a modern systems theory approach. Courier Corporation, Chelmsford

6. Tsai TH, Hussain MA (2020) VLSI implementation of lossless ecg compression algorithm for low power devices. IEEE Trans Circuits Syst II Express Briefs 67(12):3317-3321

7. Qu C, Zhu Z, En Y, Wang L, Liu X (2020) Area-efficient extended 3-D inductor based on TSV technology for RF applications. IEEE Trans Very Large Scale Integr(VLSI) Syst 29(2):287-296
8. Zito D, Pepe D, Fonte A (2014) High-frequency CMOS active inductor: design methodology and noise analysis. IEEE Trans Very Large Scale Integr(VLSI) Syst 23(6):1123-1136

9. Gawronski W (2006) Balanced control of flexible structures, vol 211. Springer, Berlin

10. Yin Z, Xiong J (2018) Discrete-time lossless positive real lemma based on Kalman reachability decomposition In: 2018 15th international conference on control, automation, robotics and vision (ICARCV), pp 662-667

11. Kothyari A, Praagman C, Belur MN (2018) Lossless systems storage function: new results and numerically stable and non-iterative computational methods. IEEE Trans Circ Syst 65:4349-4362

12. Sandberg H, Delvenne JC, Doyle JC (2011) On lossless approximations, the fluctuation-dissipation theorem, and limitations of measurements. IEEE Autom Control 56:293-308

13. Figotin A, Welters A (2012) Dissipative properties of systems composed of high-loss and lossless components. J Math Phys 53:123508

14. Anderson B (1968) A simplified viewpoint of hyperstability. IEEE Trans Autom Control 13:292-294

15. Hewer G, Kenney C (1987) Dissipative LQG control systems. In: 26th IEEE conference on decision and control, Vol. 26, pp 939-944, December

16. Buscarino A, Fortuna L, Frasca M, Xibilia MG (2012) An analytical approach to one-parameter MIMO systems passivity enforcement. Int J Control 85:1235-1247

17. Anderson BD, Vongpanitlerd S (2013) Network analysis and synthesis: a modern systems theory approach. Courier Corporation, Chelmsford

18. Willems JC (1976) Realization of systems with internal passivity and symmetry constraints. J Franklin Inst 301(6):605-621

19. Hitz L, Anderson BDO (1969) Discrete positive-real functions and their application to system stability. In: Proceedings of the Institution of Electrical Engineers, Vol. 116

20. Xiao C, Hill DJ (1999) Generalizations and new proof of the discrete-time positive real lemma and bounded real lemma. IEEE Trans Circuits Syst I Fundam Theory Appl 46(6):740-743

21. Fortuna L, Frasca M (Eds.) (2012) Optimal and robust control: advanced topics with MATLAB R. CRC Press

22. Lancaster P, Rodman L (1995) Algebraic Riccati equations. Clarendon Press, Chelmsford

23. Lozano-Leal R, Joshi SM (1988) On the design of the dissipative LQG-type controllers. In: Proceedings of the 27th IEEE conference on decision and control, pp 1645-1646. IEEE

24. Jonckheere EA (1983) A Closed-loop principal component analysis of a tetrahedral truss. In: Proceedings of the workshop on applications of distributed system theory to the control of large space structures, p 163 